\title{
COMPRESSIVE SENSING FOR NEUTROSPHERIC WATER VAPOR TOMOGRAPHY USING GNSS AND INSAR OBSERVATIONS
}

\author{
${ }^{1,2}$ Marion Heublein, ${ }^{1,3}$ Xiao Xiang Zhu, ${ }^{2}$ Fadwa Alshawaf, ${ }^{4}$ Michael Mayer, ${ }^{1,3}$ Richard Bamler, ${ }^{2}$ Stefan Hinz \\ ${ }^{1}$ Remote Sensing Technology Institute, German Aerospace Center (DLR), Oberpfaffenhofen, Germany \\ ${ }^{2}$ Institute of Photogrammetry and Remote Sensing, Karlsruhe Institute of Technology, Germany \\ ${ }^{3}$ Chair of Remote Sensing Technology, Technische Universität München, Germany \\ ${ }^{4}$ Geodetic Institute, Karlsruhe Institute of Technology, Germany
}

\begin{abstract}
This paper presents the innovative Compressive Sensing (CS) concept for tomographic reconstruction of 3D neutrospheric water vapor fields using data from Global Navigation Satellite Systems (GNSS) and Interferometric Synthetic Aperture Radar (InSAR). The Precipitable Water Vapor (PWV) input data are derived from simulations of the Weather Research and Forecasting modeling system. We apply a Compressive Sensing based approach for tomographic inversion. Using the Cosine transform, a sparse representation of the water vapor field is obtained. The new aspects of this work include both the combination of GNSS and InSAR data for water vapor tomography and the sophisticated CS estimation: The combination of GNSS and InSAR data shows a significant improvement in 3D water vapor reconstruction; and the CS estimation produces better results than a traditional Tikhonov regularization with $l_{2}$ norm penalty term.
\end{abstract}

Index Terms-Atmospheric modeling, tomographic reconstruction, Compressive Sensing, GNSS, InSAR

\section{MOTIVATION AND INTRODUCTION}

High precision applications using space-geodetic techniques, such as GNSS (Global Navigation Satellite Systems) and InSAR (Interferometric Synthetic Aperture Radar), require a complete modeling of neutrospheric effects. On their propagation through the Earth's atmosphere, the radio-wave signals are affected by neutrospheric water vapor. Therefore, methods have been developed to accurately determine the neutrospheric water vapor content from the GNSS as well as InSAR observations. Various research have been carried out since [1] introduced GNSS meteorology as an important tool for determining the spatially and temporally highly variable neutrospheric water vapor content.

InSAR data do not produce absolute water vapor information. In contrast, partial wet delays can be deduced from the differential InSAR neutrospheric phase observations. An elevation-dependent as well as a long-wavelength part of the wet delay are removed within the InSAR data processing [2].
For neutrospheric modeling, these components have to be reconstructed using dense GNSS precipitable water vapor (PWV) measurements as described in [2]. The PWV deduced from GNSS and InSAR wet delays only yield integrated 2D information of the neutrospheric water vapor content. This calls for tomographic approaches that reconstruct 3D water vapor fields from GNSS and InSAR wet delay measurements. The main challenge for the tomographic reconstruction of water vapor is the limited number of measurements. Due to the pointwise observing geometry of GNSS, many voxels remain undetermined, particularly those in lower neutrospheric layers. In order to solve the underdetermined and ill-conditioned inverse problem, generalized inverse matrices resulting from singular value decomposition, constraints using a priori knowledge resp. fixing the behavior of neighboring voxels, or additional information are necessary.

In this paper, we exploit the fact that neutrospheric water vapor mixing ratios can be sparsely represented in an orthogonal basis, for instance the Cosine transform matrix, as a strong prior to regularize the addressed underdetermined problem.

\section{SENSOR CHARACTERISTICS AND DATASETS}

Both GNSS and InSAR dispose of an all-weather observing capability. Using the method of Precise Point Positioning described in [3], GNSS yield point-wise estimates of the integrated slant wet delay caused by neutrospheric water vapor. The spatial resolution depends on the density of the observing sites, and as illustrated in Figure 1, each estimated value represents the neutrospheric effect within a cone with a vertex at the GNSS site. GNSS observations of ten sites within a $100 \mathrm{~km} \times 100 \mathrm{~km}$ area in the Upper Rhine Graben in southern Germany are available at a temporal resolution of 30 seconds. In contrast, InSAR neutrospheric phase maps derived from acquisitions of the C-band Envisat satellite are available at 35 days repeat cycles. However, the spatial resolution of InSAR is significantly high, i.e. up to $100 \mathrm{~m}$. The InSAR data processing was done using the Persistent Scatterer Interferometry (PSI) introduced by [4]. At this stage, we only use sensor geometries to better evaluate the proposed approach: We are working with simulated data in this preliminary study. 


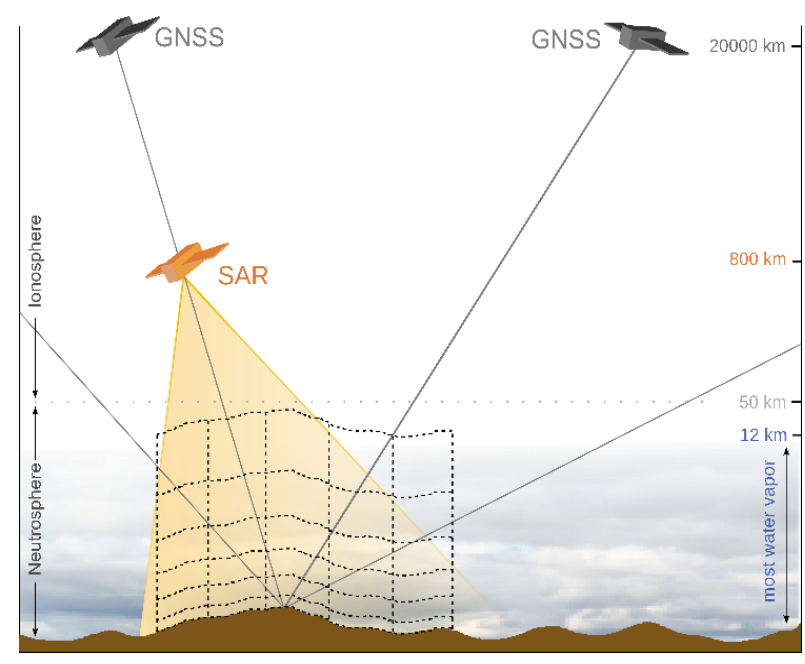

Figure 1: Observing geometry of GNSS and InSAR

Namely, 3D water vapor fields with a resolution of $900 \mathrm{~m}$ in longitude and latitude are simulated using the Weather Research and Forecasting (WRF) modeling system. Based on the observing geometry of the available GNSS and InSAR measurements, integrated GNSS and InSAR data are calculated from the WRF data. This enables a direct comparison of the later estimated 3D water vapor field with the reference data available from WRF. Moreover, in doing so, a study of the minimum number of GNSS sites required for a reasonable $3 \mathrm{D}$ water vapor reconstruction becomes possible. Figure 2 summarizes the relation between GNSS or InSAR integrated wet delays (or PWV) and the 3D water vapor mixing ratios simulated by WRF.

\section{METHODOLOGY}

In order to explain the developed methodology, this section first introduces the system model. Then, Tikhonov regularization [5], a Compressive Sensing (CS) regularization benefitting of the sparsity of the signal, and the SL1MMER algorithm combining CS and least squares [6] are explained. In addition, important parameters for CS are defined.

\subsection{System model}

The neutrospheric test region in the URG is subdivided into $p \times q=5 \times 5$ pixels equidistant in longitude and latitude. The thickness of the $r=7$ height layers increases with height (from Earth surface to top boundary in m: 350, 500, 750, $1000,1500,2500,3400)$. An upper boundary layer of $10 \mathrm{~km}$ has been chosen for tomographic reconstruction. WRFderived GNSS and InSAR wet delay maps $y \in \mathbb{R}^{N \times 1}$ result from the linear measurement process represented by the matrix $\Phi \in \mathbb{R}^{N \times L}$ in

$$
y=\Phi x+\varepsilon
$$

The parameter $x \in \mathbb{R}^{L \times 1}$ contains the unknown water vapor mixing ratios for each of the $L=p \times q \times r$ volume pixels (voxels), $\varepsilon$ is noise. A total of $N$ observations are available. If a GNSS or InSAR ray crosses a voxel, the entry $\varphi_{i j}$ of $\Phi$ equals the crossed distance within the respective voxel $j$ for the $i^{\text {th }}$ observation; otherwise it is zero. The distances are computed using the Smits' algorithm [7]. For simplification, i) no bending of the ray path is assumed, ii) the density of dry air is set to $1 \mathrm{~kg} / \mathrm{m}^{3}$, and iii) specific humidity and water vapor mixing ratio are assumed equal in a first approximation. The iii) simplification can be done because the water vapor mixing ratios

$$
w_{v}=\frac{m_{W V}}{m_{\text {dry air }}}
$$

in $[\mathrm{kg} / \mathrm{kg}]$ are close to zero in cold regions and can be transformed into values of the specific humidity $q_{v}$ by means of

$$
q_{v}=\frac{w_{v}}{1+w_{v}} \approx w_{v}
$$

In equation (2), $m_{W V}$ resp. $m_{\text {dry air }}$ represent the mass of water vapor resp. of dry air.

Reconstructing 3D neutrospheric water vapor fields from GNSS and InSAR data requires solving the linear system of equations given in (1). According to classical adjustment theory, a unique solution can be found if the number of observations is at least as large as the number of unknown parameters. However, using GNSS observations and only a single InSAR swath, many voxels remain undetermined in the lower neutrospheric layers. Voxels that are crossed by multiple rays cannot contribute to voxels without any observations. As a result, the system (1) is underdetermined and ill-conditioned and has to be regularized in order to yield a unique solution.

\subsection{Solution using Tikhonov regularization}

The generalized Tikhonov regularization incorporates a side constraint into (1) and determines the solution with minimum $l_{2}$ norm:

$$
\hat{x}_{\lambda}=\operatorname{argmin}\left\{\|\Phi x-y\|_{2}^{2}+\lambda\left\|M\left(x-x_{0}\right)\right\|_{2}^{2}\right\}
$$

The left term in (4) is an $l_{2}$ norm residual term; the right term represents the $l_{2}$ norm of the unknowns. The parameter $\lambda$ provides a trade-off between these two terms. The matrix $M$ corresponds, e.g., to the identity matrix, and $x_{0}$ contains prior information on the parameters. If no prior information is available, $x_{0}$ is set to zero.

\subsection{Solution using Compressive Sensing}

A signal is sparse if the signal itself or a transformed version after projecting to a certain basis contains only a small number of non-zeros. A sparse representation $s \in \mathbb{R}^{L \times 1}$ of the above described water vapor mixing ratios is obtained in an appropriate basis by a linear transform $s=\Psi x$ resp. $x=\Psi^{T} S$ resulting in 


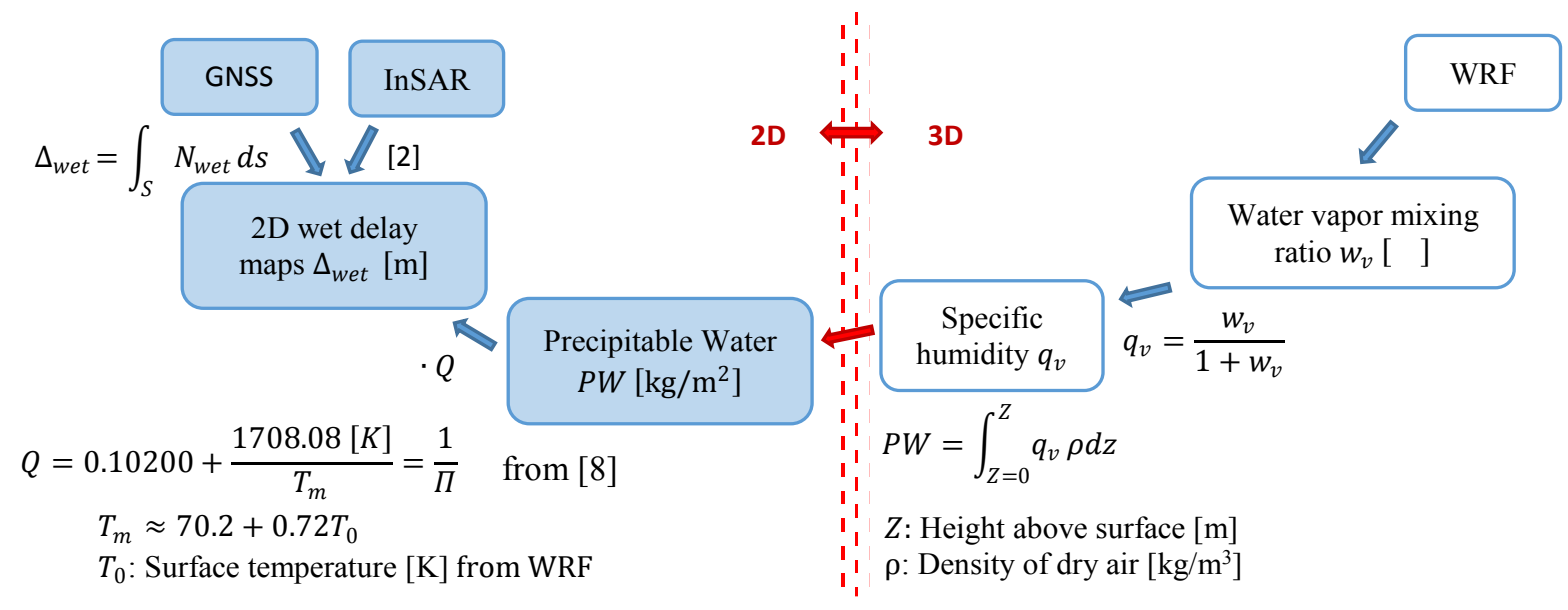

Figure 2: 2D wet delay maps from GNSS and InSAR vs. 3D water vapor mixing ratios from WRF

$$
y=\Phi \Psi^{T} s+\varepsilon=\Theta s+\varepsilon .
$$

The mapping matrix $\Theta=\Phi \Psi^{T} \in \mathbb{R}^{N \times L}$ is composed of the design matrix $\Phi$ and the $3 \mathrm{D}$ discrete Cosine transform matrix $\Psi \in \mathbb{R}^{L \times L}$ computed according to [9]. The Cosine transform yields a sparse representation of the water vapor signal. The CS inversion is then solved by minimizing the $l_{0}$ norm, i.e. the number of non-zero entries of the solution $s$ is minimized. However, this solution is non-deterministic polynomial-time hard (NP-hard). Therefore, [10] proposes a signal recovery by means of the $l_{1}$ norm regularization

$$
\hat{s}=\operatorname{argmin}\|s\|_{1} \text { such that }\|\Theta s-y\| \leq \varepsilon .
$$

\subsection{SL1MMER algorithm}

In a first step, the SL1MMER algorithm introduced by [6] uses (6) to estimate the sparse coefficients of the considered signal. However, due to the non-stable mapping matrix in tomographic water vapor sensing, CS often overestimates the sparsity of the signal, and outliers are contained in the sparse coefficients. Therefore, a careful model selection is applied in order to derive more accurate estimates of the number of non-zero coefficients and their positions within $\hat{s}$. Finally, the mapping matrix $\Theta$ is reduced to

$$
\Theta_{\text {slim }}=\Theta_{[k]}
$$

where $[k]$ represents the set of indices corresponding to the non-zero sparse coefficients and $\Theta_{[k]}$ is the corresponding set of columns of $\Theta$. Only those columns remain for the final least squares estimation of the sparse parameters

$$
\hat{s}_{[k]}=\left(\Theta_{[k]}^{T} \Theta_{[k]}\right)^{-1} \Theta_{[k]} y .
$$

\subsection{Analysis on sparsity, incoherence, and RIP in CS}

In order to obtain an accurate solution with high probability from (6), the signal $x$ must be sparsely represented by
$s=\Psi x$, the design matrix $\Phi$ and the representation matrix $\Psi^{T}$ have to be incoherent, and the restricted isometry constant $\delta_{K}$ should be low, see [10], [11].

Sparsity analyses based on simulations of the WRF modeling system show that the Cosine transform, when compared to Fourier, biorthogonal 3.7 wavelet, and curvelet transforms, yields the sparsest representation of the 3D water vapor field. More than $99 \%$ of the signal power is contained in less than $2 \%$ of the coefficients. The coherence $\mu \in[1, \sqrt{L}]$ between $\Phi$ and $\Psi^{T}$ is defined as

$$
\mu\left(\Phi, \Psi^{T}\right)=\sqrt{L} \max _{1 \leq m, n \leq L} \frac{\left|\left\langle\varphi_{m}, \psi_{n}^{T}\right\rangle\right|}{\left\|\varphi_{m}\right\|_{2}\left\|\psi_{n}^{T}\right\|_{2}}
$$

where $\varphi_{m}$ and $\psi_{n}^{T}$ represent the rows of $\Phi$ and $\Psi^{T}$. For $L=5 \times 5 \times 7$, the coherence can reach values within $\mu \in[0, \sqrt{175}] \approx[0,13.23]$. In case of the Cosine transform, the values $\mu \approx 7.65$ to 8.25 (GNSS and InSAR) resp. $\mu \approx 5.36$ to 5.95 (GNSS only) are obtained if 10 to 30 GNSS sites are introduced into the processing. In [10], the restricted isometry constant is defined as the smallest $\delta_{K} \geq 0$ satisfying

$$
\left(1-\delta_{K}\right)\|v\|_{2}^{2} \leq\|\Theta v\|_{2}^{2} \leq\left(1+\delta_{K}\right)\|v\|_{2}^{2}
$$

for all $K$-sparse vectors $v$ containing $K$ non-zero elements. According to [11], $K$-sparse signals can be exactly (resp. stably) reconstructed via $l_{1}$ minimization if the restricted isometry property, short RIP, is fulfilled, i.e., if $\delta_{K}<0.307$ in the absence (resp. in presence) of noise.

\section{RESULTS AND DISCUSSION}

Although $\delta_{K}>0.307$ in this study, the accuracy of the estimated water vapor mixing ratios is significantly improved by using CS or SL1MMER (which utilized sparsity as a prior) when compared to traditional $l_{2}$ norm minimization without prior. Moreover, as shown in Figure 3, using both GNSS and InSAR data is profitable. The quantity 

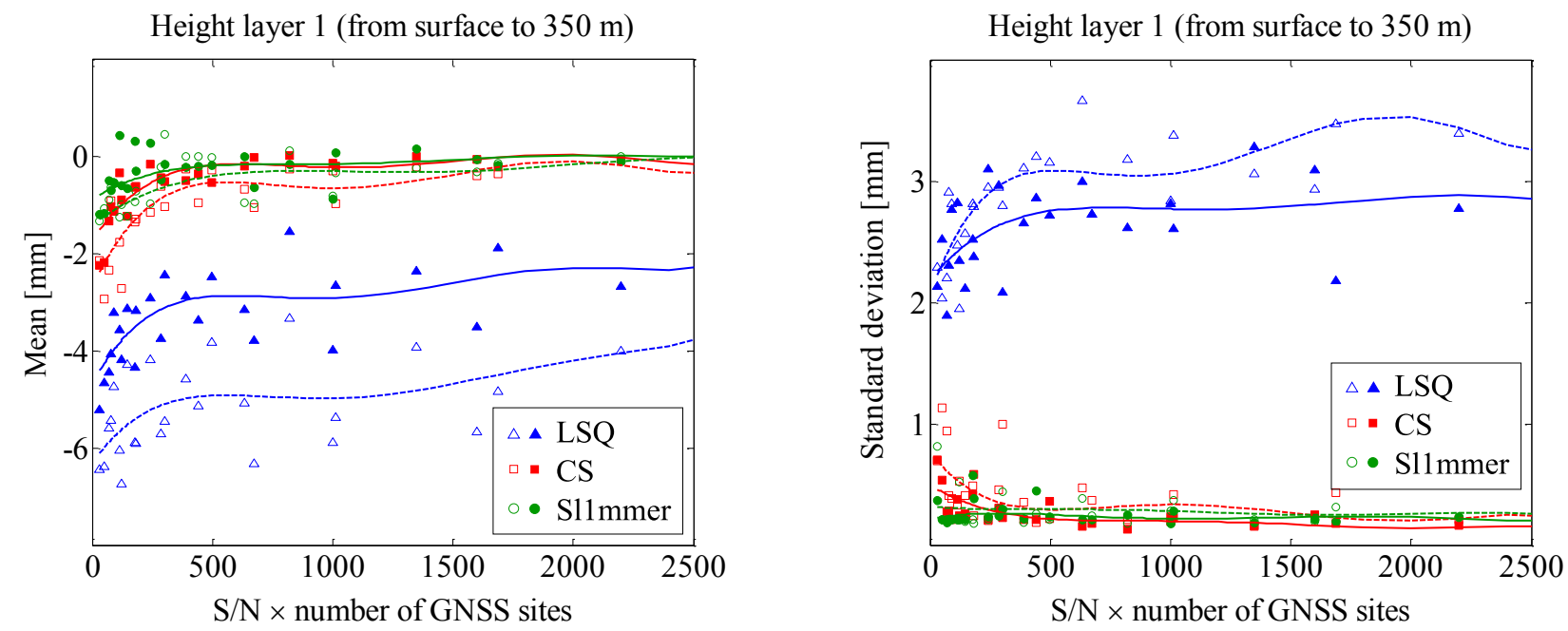

Figure 3: Mean and standard deviation of wet delay differences (estimated - true) on January 3, $2005 \mathrm{in} \mathrm{mm}$, with polynomial fit of degree 5. Filled (resp. empty) markers and solid (resp. dotted) lines correspond to data derived from GNSS and InSAR (resp. GNSS only). The LSQ data are computed according to section 3.1 without prior information. The CS data result from section 3.2, SL1MMER data are derived according to section 3.3. The mean wet delay at heights up to $350 \mathrm{~m}$ is about $8.2 \mathrm{~mm}$.

$$
n=\mathrm{S} / \mathrm{N} \times \text { number of GNSS sites }
$$

should exceed $n=350$ in order to get precise and accurate results. Assuming a constant SNR of $25 \mathrm{~dB}(\mathrm{~S} / \mathrm{N} \approx 17.8)$, at least 20 GNSS sites should be included into our analysis.

The current horizontal resolution of $20 \mathrm{~km}$ has been chosen because of the small number of GNSS sites in the considered region and in order to check the CS approach in a computationally reasonable way. However, for higher resolved neutrospheric modeling and further research, smaller voxel sizes of about $10 \mathrm{~km}$ are under focus. Realistic values of SNR should be analyzed and it has to be kept in mind that SNR will not be constant for wet delay observations along different elevation and azimuth angles as well as in case of multipath or obstacles.

This study presented a concept for CS based tomographic reconstruction of neutrospheric water vapor. As the RIP is violated in this analysis, the probability to accurately reconstruct the signal is low. Therefore, the estimation should be performed repeatedly and the results of the different runs should be compared. Real data will replace the current wet delay simulations from WRF.

\section{Acknowledgements}

Many thanks go to Franz Ulmer (Remote Sensing Technology Institute, German Aerospace Center) and to Benjamin Fersch (Institute of Meteorology and Climate Research, Karlsruhe Institute of Technology) for providing WRF simulations for this analysis.

\section{REFERENCES}

[1] M. Bevis, S. Businger, T. A. Herring, C. Rocken, R. A. Anthes, and R. H. Ware, "GPS Meteorology: Remote Sensing of
Atmospheric Water Vapor using the Global Positioning System," Journal of Geophysical Research: Atmospheres, 97(D14), pp. 15787-15801, 1992.

[2] F. Alshawaf, S. Hinz, M. Mayer, and F. Meyer, "Constructing accurate maps of atmospheric water vapor by combining interferometric synthetic aperture radar and GNSS observations," Journal of Geophysical Research: Atmospheres, 120(4), pp. 1391-1403, 2015.

[3] J. Kouba and P. Héroux, "GPS Precise Point Positioning using IGS Orbit and Clock Products," GPS Solutions, 5(2), pp. 1228, 2000.

[4] A. Hooper, P. Segall, and H. Zebker, "Persistent scatterer interferometric synthetic aperture radar for crustal deformation analysis, with application to Volcán Alcedo, Galápagos," Journal of Geophysical Research: Solid Earth, 112(B7), 2007.

[5] D. Calvetti and L. Reichel, "Tikhonov regularization with a solution constraint," SIAM Journal on Scientific Computing, 26, pp. 224-239, 2004.

[6] X. X. Zhu and R. Bamler, "Compressive sensing for high resolution differential SAR tomography - the SL1MMER algorithm," IEEE International Geoscience and Remote Sensing Symposium, pp. 17-20, 2010.

[7] B. Smits, "Efficiency issues for ray tracing," Journal of Graphics Tools, 3(2), pp. 1-14, 1999.

[8] T. Schüler, "On ground-based GPS tropospheric delay estimation," Universität der Bundeswehr München, 2001.

[9] Y.-L. Chan and W.-C. Siu, "Variable Temporal-Length 3-D Discrete Cosine Transform Coding," IEEE Transactions on Image Processing, 6(5), pp. 758-763, 1997.

[10] E. Candès and M. B. Wakin, "An Introduction to Compressive Sampling," IEEE Signal Processsing Magazine, 25(2), pp. 2130, 2008.

[11] T. T. Cai, L. Wang, and G. Xu, "New bounds for restricted isometry constants," IEEE Transactions on Information Theory, 56(9), pp. 4388-4394, 2010. 